\title{
Baclofen-induced Changes in the Resting Brain Modulate Smoking Cue Reactivity: A Double-blind Placebo-controlled Functional Magnetic Resonance Imaging Study in Cigarette Smokers
}

\author{
Ariel Ketcherside ${ }^{1}$, Kanchana Jagannathan ${ }^{1}$, Sudipto Dolui ${ }^{1}$, Nathan Hager ${ }^{1,2}$, Nathaniel Spilka ${ }^{1}$, Chaela Nutor ${ }^{1}$, \\ Hengyi Rao', Teresa Franklin ${ }^{1, *}$, Reagan Wetherill ${ }^{1} *$ \\ ${ }^{1}$ The Center for Studies of Addiction, The University of Pennsylvania Perelman School of Medicine, Philadelphia, PA, ${ }^{2}$ Department of Psychology, \\ Old Dominion University, Norfolk, VA, USA
}

\begin{abstract}
Objective: Smoking cue-(SC) elicited craving can lead to relapse in SC-vulnerable individuals. Thus, identifying treatments that target SC-elicited craving is a top research priority. Reduced drug cue neural activity is associated with recovery and is marked by a profile of greater tonic (resting) activation in executive control regions, and increased connectivity between executive and salience regions. Evidence suggests the GABA-B agonist baclofen can reduce drug cue-elicited neural activity, potentially through its actions on the resting brain. Based on the literature, we hypothesize that baclofen's effects in the resting brain can predict its effects during SC exposure.

Methods: In this longitudinal, double blind, placebo-controlled neuropharmacological study 43 non-abstinent, sated treatment-seeking cigarette smokers $(63 \%$ male) participated in an fMRI resting-state scan and a SC-reactivity task prior to (T1) and 3 weeks following randomization ( $T 2$; baclofen: $80 \mathrm{mg} /$ day; $\mathrm{n}=21$ ). Subjective craving reports were acquired before and after SC exposure to explicitly examine SC-induced craving.

Results: Whole-brain full-factorial analysis revealed a group-by-time interaction with greater resting brain activation of the right dorsolateral prefrontal cortex (dIPFC) at T2 in the baclofen group (BAC) $\left(p_{\text {FWEcorr }}=0.02\right)$, which was associated with reduced neural responses to SCs in key cue-reactive brain regions; the anterior ventral insula and ventromedial prefrontal cortex $\left(p_{\text {FWEcorr }}<0.01\right)$. BAC, but not the placebo group reported decreased SC-elicited craving $(p=0.02)$. Conclusion: Results suggest that baclofen mitigates the reward response to SCs through an increase in tonic activation of the dIPFC, an executive control region. Through these mechanisms, baclofen may offer SC-vulnerable smokers protection from SC-induced relapse.
\end{abstract}

KEY WORDS: Baclofen; Cerebral blood flow; Cue-elicited craving; Nicotine; Perfusion fMRI; Resting-state.

\section{INTRODUCTION}

The consequences of smoking are devastating, yet approximately $20 \%$ of the world's population above the age of 14 continues to smoke [1]. Although smokers indicate that they want to quit, success rates are low [2], and over

Received: August 7, 2019/ Revised: November 1, 2019 Accepted: January 4, 2020

Address for correspondence: Ariel Ketcherside

The Center for Studies of Addiction, The University of Pennsylvania Perelman School of Medicine, 3535 Market St. Ste 500, Philadelphia, PA 19104, USA

E-mail: Ariel.Ketcherside@gmail.com

ORCID: https://orcid.org/0000-0003-1885-3581

*These authors contributed equally to this study as co-senior authors. half of those who have made a quit attempt will relapse within a year [3]. Because of the myriad caveats associated with the study of real-world smoking, the role of smoking cue (SC) exposure in relapse remains equivocal [4]. However, SC-elicited craving persists as a key metric by which to assess the severity of nicotine use disorder because cues elicit conditioned responses such as craving and approach behavior [5-7]. Further, former smokers continue to view SCs as salient long after cessation, and often cite SC exposure as their reason for relapse [8].

SCs increase neural activity in reward-related brain regions, independent of pharmacological withdrawal [9], SC-induced craving can predict increased smoking behavior [10], and SC-elicited neural activation in reward

(c) This is an Open-Access article distributed under the terms of the Creative Commons Attribution Non-Commercial License (http://creativecommons.org/licenses/by-nc/4.0) which permits unrestricted non-commercial use, distribution, and reproduction in any medium, provided the original work is properly cited. 
regions can predict relapse [11]. Furthermore, decreased functional connectivity between a salience region (anterior ventral insula; avInsula) and an executive control region (the dorsolateral prefrontal cortex; dIPFC) during SC exposure predicts relapse, indicating that decoupling of salience and executive control regions contribute to SC vulnerability [12]. Similarly, smokers with greater response in reward regions during SC exposure have greater difficulty quitting smoking [13]. Collectively, these studies elucidate a select role of SC vulnerability in relapse.

The primary smoking cessation medications are nicotine replacement therapy, bupropion, and varenicline, all three of which reduce withdrawal from nicotine [14]. Varenicline, the most effective and most commonly prescribed, has a second mechanism: before cessation, it blocks nicotine's reinforcing effects $[15,16]$. Nonetheless, varenicline does not help most individuals to whom it is prescribed, as relapse rates remain high [17]. In line with varenicline's mechanism, varenicline-responders could represent a subgroup of smokers whose vulnerability to relapse is coupled with nicotine reinforcement, while those who do not respond to varenicline may have unmet vulnerabilities such as those associated with SC exposure. Currently, there are no medications directly targeting SC reactivity to aid SC-vulnerable individuals.

GABA-B agonists inhibit dopaminergic afferents in reward regions, including nicotine-induced reward signaling [18]. There is substantial evidence that the GABA-B agonist baclofen blocks conditioned drug-related behaviors and may target drug cue vulnerabilities in humans [19-22]. One functional magnetic resonance imaging (fMRI) study showed that baclofen reduced reward-related neural responses to cocaine cues in abstinent, treatment-seeking cocaine participants, however baclofen-induced changes from baseline could not be determined from that study [23]. Another recent neuropharmacological study examined baclofen's effects on alcohol cue reactivity in abstinent alcohol use disorder (AUD) treatment-seeking patients relapse [24]. Authors examined cue reactivity prior to and after two weeks of medication. Baclofen increased activity of the anterior cingulate cortex (ACC) and bilateral dIPFC while decreasing responses in the ventral striatum (VS) and orbitofrontal and insular cortices. Responses in the ACC and insula predicted time to relapse [24]. Collectively, these studies suggest a common mechanism whereby baclofen blunts the neural response to drug cues, which could be a useful/necessary mechanism of blunting cue-induced craving in cue-vulnerable substance use disorder patients.

Using the quantitative technique of perfusion $\mathrm{fMRI}$, we have shown that baclofen increases cerebral blood flow $(\mathrm{CBF})$ in executive control regions, including the dIPFC and $A C C$, in the resting brain and blunts $C B F$ in reward regions, suggesting tonic modulation of the brain at rest [25]. Greater tonic activation of executive control regions, as operationalized by resting state neuroimaging, could be an indicator of nicotine use disorder improvement: greater tonic executive control may be necessary to avoid a lapse when encountering SCs [26-28].

In the present study, we seek to extend the findings of ourselves and others by determining whether baclofen-related changes in the resting brain are associated with SC-reactivity within cigarette smokers. Based on our previous findings and those of Holla et al. [24], 2018 and Janes et al. [12], 2010, described above, we hypothesized (1) that baclofen would increase $C B F$ in the bilateral dIPFC and the ACC in the resting brain, and (2) that the increases observed in resting CBF would be associated with decreases in $\mathrm{CBF}$ during $\mathrm{SC}$ exposure in our a priori reward regions (i.e., ventromedial prefrontal cortex/medial orbitofrontal cortex [vmPFC/mOFC], VS, and avInsula) $[9,11,12,24,29,30]$.

\section{METHODS}

All procedures were conducted at the University of Pennsylvania Center for Studies of Addiction. All procedures were approved by the University of Pennsylvania's Institutional review board (IRB No. 817101) and conducted in accordance with the Declaration of Helsinki. All participants provided written informed consent. This study was pre-registered at ClinicalTrials.gov, with Identifier: NCT01821560.

\section{Participants}

Participants were treatment-seeking cigarette smokers, 18-60 years old, who smoked at least 10 cigarettes per day (CPD) for the past 6 months. Interested volunteers were recruited by flyers, listservs, and word of mouth and completed an initial phone screen and, if eligible, an in-person consent and screening visit. After providing informed consent, participants completed the Fagerström 
Test for Nicotine Dependence (FTND) [31] and underwent physical and psychiatric screening. Physical screening included a blood test to ensure general physical health, an EKG, a urine toxicology test to rule out the presence of psychoactive drugs, and a pregnancy test in females. Psychological screening ascertained any current severe psychiatric symptoms, or diagnosis of any substance use disorders (SUDs) other than nicotine (MINI; [32]). Participants were excluded for current other SUDs, current Axis 1 DSM-IV disorder, an intellectual ability estimate score below 80 [33], use of any other smoking cessation strategies, and any MRI contraindications [34]. Potential contraindications were reviewed by the study physician, and participants were financially compensated for participation. Participants were randomized to receive either baclofen or placebo after their first MRI scan. The study design is illustrated in Figure 1.

Twenty-three baclofen recipients (BAC) and 22 placebo recipients $(\mathrm{PBO})$ completed study procedures. One participant was excluded for abnormally large ventricles and one participant was excluded for insufficient signal-to-noise ratio in their resting-state data.

Subjective craving scores were excluded for two baclofen participants based on an error in assessment administration. Analyses were completed on the remaining 21
BAC and 22 PBO, except the SC-elicited craving analyses which included 19 BAC and 22 PBO. Participant characteristics are listed in Table 1.

\section{Medication}

Study medication was manufactured and provided by Murty Pharmaceuticals, Inc., Lexington, KY, USA. The Investigational Drug Service at the University of Pennsylvania maintained and distributed medication in blister packs (randomized by medication group and sex). All study staff and participants were blinded to the medication administered. A nurse practitioner met with participants weekly to collect used pill packs (compensation was provided to encourage adherence), distribute new packs, and monitor adverse events and medication adherence/issues. The most common adverse event reported for baclofen is drowsiness. Based on pilot data conducted in our lab, we were able to accelerate the titration schedule used in our previous study [25] without affecting side effects, including drowsiness. Similarly to Logge et al. [22], 2019, we used a titration schedule wherein participants reached the full dose of $80 \mathrm{mg}$ ( $20 \mathrm{mg}$ q.i.d.) by day 4 (Table 2).

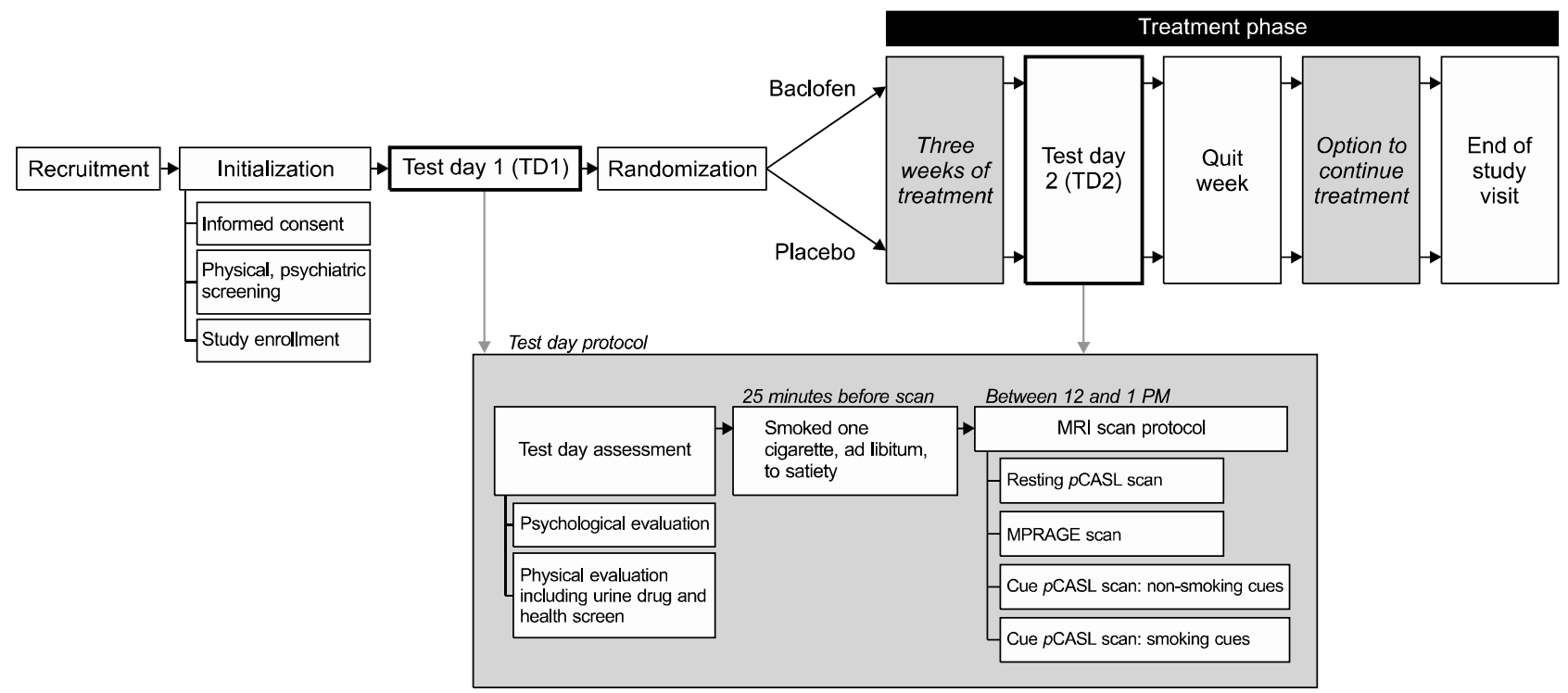

Fig. 1. Study design. Participants underwent a double-blind, placebo-controlled study in which they received 20 mg q.i.d. baclofen or placebo. They participated in a functional magnetic resonance imaging (fMRI) session before randomization (T1), and a second session after three weeks of treatment (T2). At each session, one of two similarly valanced smoking cue (SC) and non-SC sets was presented in a pseudorandomized fashion to control for habituation. $p$ CASL, pseudo-continuous arterial-spin labeled; MPRAGE, magnetization-prepared rapid gradient echo. 
Table 1. Demographics and smoking characteristics

\begin{tabular}{|c|c|c|c|c|}
\hline \multirow{2}{*}{ Variable } & \multirow{2}{*}{ Baclofen $(n=21)$} & \multirow{2}{*}{ Placebo $(n=22)$} & \multicolumn{2}{|c|}{ Group comparisons } \\
\hline & & & $t$ or $\chi^{2}$ & $p$ value \\
\hline Sex, male & $13(62)$ & $14(64)$ & 0.01 & 0.91 \\
\hline Age & $38.9 \pm 12$ & $40.2 \pm 12$ & -0.47 & 0.64 \\
\hline IQ & $97.4 \pm 12.1$ & $103.8 \pm 12.4$ & -1.64 & 0.11 \\
\hline \multicolumn{5}{|l|}{ Race } \\
\hline African American & $12(55)$ & $14(64)$ & 0.19 & 0.66 \\
\hline Caucasian & $7(33)$ & $6(27)$ & 0.19 & 0.67 \\
\hline Asian & $2(10)$ & $2(9)$ & $<0.01$ & 0.96 \\
\hline \multicolumn{5}{|l|}{ Ethnicity } \\
\hline Hispanic & $1(0.1)$ & $3(0.1)$ & $<0.01$ & 1.0 \\
\hline Education & $13.3 \pm 1.8$ & $14 \pm 1.8$ & -1.06 & 0.29 \\
\hline \multicolumn{5}{|l|}{ Alcohol use } \\
\hline Endorsed use & $12(50)$ & $13(59.1)$ & 0.02 & 0.87 \\
\hline Drinks per drinking day, $\mathrm{T} 1^{\mathrm{a}}$ & $2.7 \pm 2.10$ & $3.1 \pm 2.4$ & 0.46 & 0.65 \\
\hline \multicolumn{5}{|l|}{ Cannabis use } \\
\hline Endorsed use & $7(31.8)$ & $8(36.4)$ & $<0.01$ & 1.0 \\
\hline Days of cannabis use, $\mathrm{T}^{\mathrm{a}}$ & $5 \pm 2.6$ & $4.5 \pm 2.4$ & 0.23 & 0.82 \\
\hline \multicolumn{5}{|l|}{ Smoking characteristics } \\
\hline FTND & $4.9 \pm 1.5$ & $4.9 \pm 1.5$ & 0.19 & 0.85 \\
\hline Pack years & $16.6 \pm 13.8$ & $15.6 \pm 12.6$ & 0.23 & 0.82 \\
\hline \multicolumn{5}{|l|}{ CPD } \\
\hline $\mathrm{T} 1$ & $14.4 \pm 5.7$ & $13.9 \pm 4.8$ & 0.35 & 0.73 \\
\hline $\mathrm{T} 2$ & $12.9 \pm 7.5$ & $9.5 \pm 4.7$ & 1.72 & 0.09 \\
\hline
\end{tabular}

Values are presented as number (\%) or mean \pm standard deviation.

T1, before treatment; T2, on medication and after 3 weeks of treatment; FTND, Fagerström Test for Nicotine Dependence; CPD, cigarettes per day. an participants who used this substance in the last 30 days, measured by the Timeline Follow Back. There were no differences between groups in any demographics or smoking characteristics.

Table 2. Baclofen titration schedule

\begin{tabular}{lcccc}
\hline \multicolumn{1}{c}{ Study day } & 8:00 AM & 12:00 PM & 4:00 PM & 8:00 PM \\
\hline Day 1 & PBO & 10 BAC & PBO & 10 BAC \\
Day 2 & PBO & 10 BAC & 10 BAC & 10 BAC \\
Day 3 & PBO & 20 BAC & 20 BAC & 20 BAC \\
Day 4-7 & 20 BAC & 20 BAC & 20 BAC & 20 BAC \\
Week 2-7 & 20 BAC & 20 BAC & 20 BAC & 20 BAC \\
Week 8 Day 1-2 & PBO & 20 BAC & 20 BAC & 20 BAC \\
Week 8 Day 3-4 & PBO & 20 BAC & PBO & 20 BAC \\
Week 8 Day 5-6 & PBO & 10 BAC & PBO & 10 BAC \\
Week 8 Day 7 & PBO & PBO & PBO & PBO \\
\hline
\end{tabular}

Baclofen (BAC) was titrated up to full dose (week 1) and tapered down (week 8) to mitigate adverse effects, including drowsiness. Placebo medication (PBO) was used to ensure blinding during the titration process.

\section{Study Design}

To determine the effect of baclofen on neural function and SC-elicited craving, we used a double-blind, placebo-controlled paradigm. At baseline, participants and study staff agreed upon a "Quit Date" on which participants would attempt to quit smoking. Importantly, this occurred after the second scan (Time 2, T2) to mitigate any effects of quitting smoking on brain endpoints. Participants were instructed to continue smoking as usual until their Quit Date. Participants began the medication regimen after their first scan (Time 1, T1). After three weeks of treatment, participants underwent a second perfusion $\mathrm{FMRI}$ scan session following the same scan protocol (T2). CPD were recorded at both timepoints. Participants then returned for their "Quit Week" appointment, at which time they received smoking cessation counseling and continued treatment for four additional weeks (Fig. 1).

All MRI sessions occurred in the early afternoon, to minimize any confounding effects of time of day, or effects of participants' daily routines on study results [35]. fMRI sessions included a pseudo-continuous arterial-spin labeled ( $p$ CASL) perfusion fMRI scan at rest and during a $\mathrm{SC}$ reactivity task. $p$ CASL is an MRI tool providing an objective measure of neural activity, using magnetically tagged blood as an inherent tracer [36]. Unlike blood oxygen level-dependent $\mathrm{fMRI}$, perfusion $\mathrm{FMRI}$ is quantitative, pro- 
viding a measure of blood flow measured in milliliters of blood/100 g of tissue/minute. This gives perfusion fMRI the unique advantage of providing a baseline measurement, and as such, it is ideal for studying the effect of pharmacological agents on neural activity [36]. Furthermore, perfusion $\mathrm{FMRI}$ is particularly suited for longitudinal studies, as it is reliable and replicable for up to seven weeks [37]. Finally, the quantitative technique of perfusion $\mathrm{fMRI}$ is ideal for measuring states that accrue slowly over time, such as craving generated by drug cues. Additionally, craving may persist after stimulus exposure, often extinguished only by smoking, thus perfusion fMRI is well-suited to our model in which stimuli are presented over several minutes.

At both timepoints, participants were non-abstinent to minimize potential confounds introduced by withdrawal and to minimize differences between groups in smoking behavior, both of which might affect neural responses. Participants smoked ad libitum throughout the day of their scan, and smoked one of their own cigarettes to satiety (ad libitum) approximately 25 minutes before data acquisition to minimize the influence of smoking on vasculature [38], which affects blood flow in the brain. Baclofen has a short half-life (approximately 4 hours), thus participants took their medication (20 mg baclofen or matching placebo) $1-1.5$ hours prior to the $\mathrm{T} 2$ session in the presence of study staff to ensure medication compliance and standardize dose time. To minimize "carryover" effects in neural response to SCs, non-SCs were presented before SCs [39]. While it is customary in some paradigms to counterbalance stimuli across scans and across participants to minimize a confounding effect of stimulus order in the results, we have previously established that (1) order effects are negligible [9], and (2) such counterbalancing can introduce a "carryover" effect in responses, as craving elicited by SCs, when presented first, are not completely extinguished by the time non-SCs are subsequently presented [39]. Participants completed the Craving and Withdrawal Questionnaire (CWQ) to assess SC-elicited changes in craving and withdrawal before and immediately after the SC task $[9,40]$.

\section{fMRI Stimuli}

Stimuli presented during the non-SC and SC scans comprised audiovisual clips, each nine minutes long, featuring actors differing in race, age, and sex, and either smok- ing-related or nonsmoking-related cues. Unlike BOLD fMRI, perfusion MRI provides an objective measurement of blood flow, which precludes the need for counterbalancing. As such, the non-SC video was presented before the SC video, to prevent carryover effects. To prevent practice effects, participants saw one of two similarly valanced SC and non-SC videos at their first scan, and the second set of videos at their second scan. The order of presentation of videos was pseudorandomized across subjects. The SC videos featured actors smoking, while using language explicitly designed to induce desire for a cigarette (e.g., "The cigarette I enjoy most is the first cigarette of the day"). The non-SC videos featured actors, but they were not smoking, and instead, told short stories unrelated to smoking and without smoking reminders. During the SC videos, subjects held one of their own cigarettes in their preferred hand, and a match was lit and extinguished, providing visual and olfactory stimuli to enhance neurophysiological and subjective cue reactivity. During the non-SC video, subjects held a freshly sharpened pencil.

\section{Scan Protocol and Parameters}

MRI scanning occurred on a Siemens 3.0 Tesla Trio whole-body scanner (Siemens AG, Erlangen, Germany), using a standard Transmit/Receive head coil. High resolution MR images were acquired using T1-weighted 3D magnetization-prepared rapid gradient echo (MPRAGE) scan (TR/TE/TI $=1,620 / 3 / 950 \mathrm{~ms}$, flip angle $=90^{\circ}$, bandwidth $=150$, voxel size $=1 \mathrm{~mm}^{3}$, matrix $=192 \times 256$, slices $=160$ ). Two-dimensional $p$ CASL perfusion $\mathrm{fMRI}$ was used to acquire resting baseline, SC, and non-SC scans. The resting scan protocol included 45 resting label/control image pairs (labeling time, 1.5 seconds; post-labeling delay, $1,000 \mathrm{~ms}$; matrix, $64 \times 64 \times 18$; flip angle, $90^{\circ}$; TR, 3.5 seconds; TE, $17 \mathrm{~ms}$; slice thickness, $7.2 \mathrm{~mm}$; voxel size $=3.44 \times 3.44 \times 7.2 \mathrm{~mm}$ ). The SC and non-SC scan protocols each included $68 \mathrm{cue}$ label/control image pairs (labeling time, 1.5 seconds; post-labeling delay, 1,500 ms; matrix, $64 \times 64 \times 18$; flip angle, $90^{\circ}$; TR, $4 \mathrm{~s}$; TE, $17 \mathrm{~ms}$; slice thickness, $7.2 \mathrm{~mm}$; voxel size $=3.44 \times 3.44 \times 7.2 \mathrm{~mm}$ ).

\section{Imaging Data Preprocessing}

We performed image processing using Statistical Parametric Mapping 12 (SPM12, the FIL Methods Group, 2016), ASL toolbox [41], Functional MRI of the Brain Software 
Library [42] and custom scripts in MATLAB 2016a [43].

Prior to the analyses, brain images were visually inspected for gross movement, structural abnormalities and whole brain coverage. Each participant's anatomical and ASL images were manually reoriented to the anterior/posterior commissure line (AC-PC line), to improve segmentation and normalization accuracy. The raw echoplanar imaging images were then realigned to correct for head motion using the method proposed by Wang [44]. The mean ASL image was aligned to the anatomical image of each subject using the FSL Boundary Based Registration (BBR) approach [45]. Using SPM, the anatomical image of each subject was probabilistically segmented into grey matter, white matter and cerebrospinal fluid to construct a binary brain mask [46]. The brain mask was down-sampled to the MNI space using the BBR co-registration parameters, and was used to restrict the $\mathrm{CBF}$ computation within the mask. Thereafter, a CBF time series was obtained by pairwise control-label subtraction, normalizing by corresponding control image, and using a single compartment model with recommended parameters [47].

\section{Image Quality Control Assessment}

A mean CBF map was obtained using a Structural Correlation with Robust Bayesian (SCRUB) estimation approach $[48,49]$ to remove artifacts from ASL-CBF images. The quality of mean CBF maps for each subject was evaluated using an automated quality evaluation index (QEI) [50], with output values between 0 (no signal) and 1 (highest signal). A QEI of 0.7 or above demonstrates high sensitivity-specificity, and thus was our criterion for inclusion in analyses. The anatomical images were normalized to the MNI space using FSL non-linear registration and this, coupled with the BBR transformation, was used to normalize the CBF maps to the MNI space. The normalized CBF maps were thereafter smoothed with a $5 \mathrm{~mm}$ full width at half maximum Gaussian kernel. For group level analyses, each model included a global calculation of the mean voxel value within the image as a regressor of no interest for data normalization.

\section{Demographics and Behavior Analyses}

We conducted all demographics and behavior analyses in R version 3.4.2 (https://www.r-project.org/) [51]. Before, and immediately after viewing SCs, participants were verbally administered the CWQ, which included a question to probe craving: "On a scale of $1-7$, with 7 as the highest and 1 as the lowest rating, how much do you crave a cigarette right now?" We calculated a change score for subjective craving (post-SC - pre-SC) such that a positive score indicates an increase in craving. We analyzed participants' responses using linear mixed models, in which subjective craving rating was the dependent variable, and group (BAC or $\mathrm{PBO}$ ), timepoint ( $\mathrm{T} 1$ or $\mathrm{T} 2$ ), and rating time (pre- or post-SC exposure) were included as fixed independent variables [52]. We included participant ID as a random variable to provide each individual's intercept. The primary result of interest was a group-by-week-bypre-/post-cue exposure interaction. We also examined each group separately via post-hoc paired $t$ tests to determine potential differences between T1 and T2 SC-elicited craving.

\section{Imaging Analyses}

As in our previous work, all analyses included participants' sex and age as nuisance covariates. No other demographic or smoking characteristics were included in the model as there were no differences between groups in any relevant variables (Table 1). All results reported in a priori regions of interest (ROIs) were small-volume corrected (SVC) with a $5 \mathrm{~mm}$ radius around the previously identified clusters $[9,25]$ and passed family-wise-error (FWE) correction, as well as a Šidák correction for multiple comparisons [53,54] at a statistical threshold of $p<0.01$. We used R and MANGO (http://ric.uthscsa.edu/mango/mango.html) [55] to generate figures.

For hypothesis 1 , that baclofen would increase CBF in cognitive control regions at rest, all participant's mean resting images were included in a full factorial analysis, to determine a group-by-timepoint interaction. Based on the literature, ROls for examining the effects of baclofen on the resting brain were the ACC $(-8,-14,40)$ and right and left dIPFC $( \pm 51,24,33)[12,24,25]$.

For hypothesis 2, that the baclofen-induced increase in ROls at rest would be associated with a decrease in the reward response during SC-exposure, we calculated a change value from the resting data (T2-T1) in the ROls and entered them independently as covariates in the SC-model at T2, as previously described [26]. Based on the literature and our significant experience examining the brain substrates of SC-induced brain activity, ROls included the VS, vmPFC, and avInsula $[9,24,29,56,57]$. 


\section{RESULTS}

\section{Participant Characteristics}

There were no differences in age, sex, race, ethnicity, education, IQ, FTND scores, CPD, pack years, or canna-

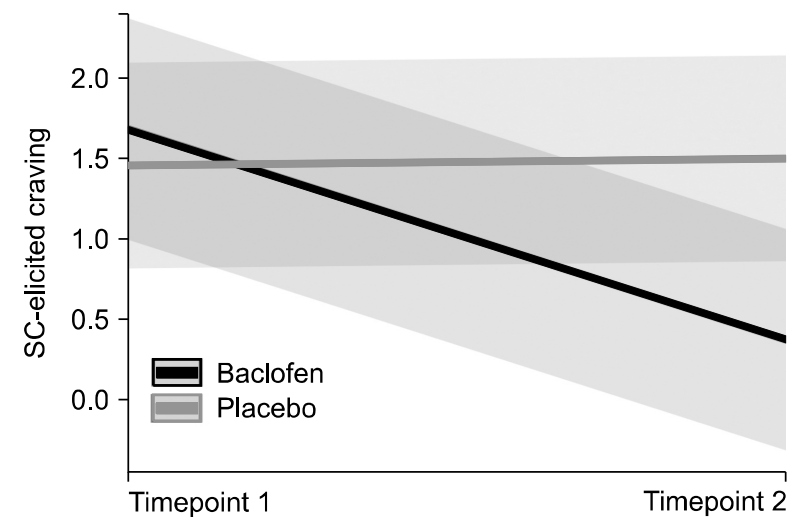

Fig. 2. Smoking cue-elicited craving before and after 3-weeks of medication. Participants were asked how much they craved a cigarette before and after seeing smoking cues. Smoking cue-induced craving scores were calculated by subtracting pre-session ratings from postsmoking cue (SC) ratings at each timepoint. Baclofen, but not placebo participants reduce SC-elicited craving from pretreatment (T1) to during treatment (T2). Translucent bars indicate 95\% confidence intervals. bis and alcohol use between $\mathrm{BAC}$ and $\mathrm{PBO}$ recipients (Table 1). A linear mixed model revealed an overall effect of CPD between the groups and timepoints, $\mathrm{F}(3,81)=$ $3.05, p=0.033$. However, there were no effects of timepoint $\beta=-1.57$, standard error (SE) $=1.78, p=0.38$, group $\beta=2.20, \mathrm{SE}=3.95, p=0.58$, or the interaction between group and timepoint, $\beta=-2.77, \mathrm{SE}=2.50, p=$ 0.27 .

\section{Adverse Events}

There were no differences between $\mathrm{BAC}$ and $\mathrm{PBO}$ groups in total number of adverse events $t(33)=1.56, p=$ $0.13, d=0.48$. Because sedation, or "drowsiness," is the adverse effect most commonly associated with baclofen, we examined it separately. There was no difference between groups in sedation, $t(36)=1.32, p=0.20, d=0.42$.

\section{Smoking Cue-elicited Craving}

Two participants' SC-elicited craving data were missing. Of the remaining participants, a linear mixed model revealed an overall effect, $\mathrm{F}(3,78)=2.95, p=0.038$. There was an effect of timepoint, $\beta=-1.32$, SE $=0.49, p<$ 0.01 , no effect of group, $\beta=-1.59, \mathrm{SE}=1.07, p=0.14$,

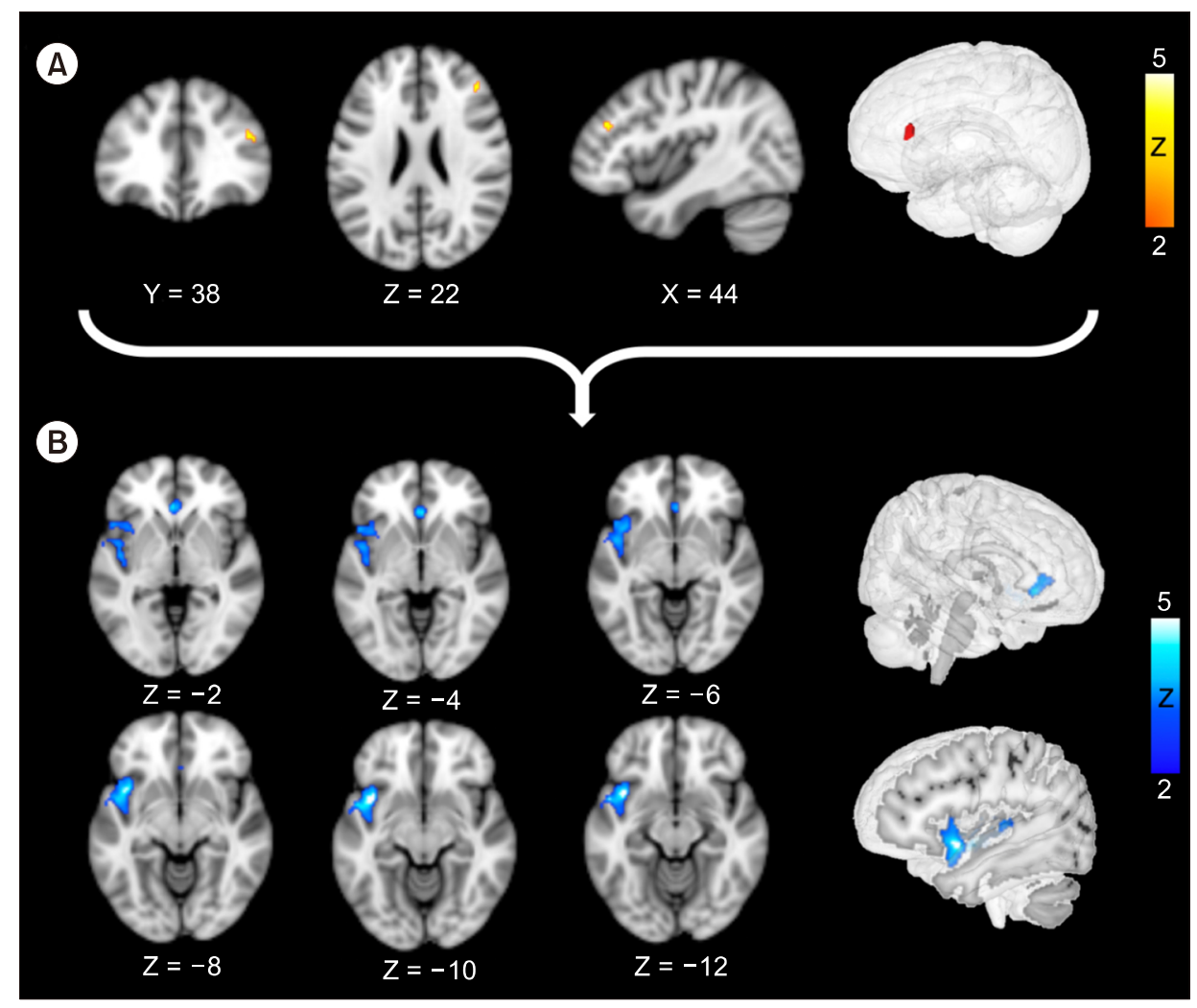

Fig. 3. The effects of baclofen on dorsolateral prefrontal cortex (dIPFC) in the Brain at Rest Predict responses to smoking cues (SCs). (A) Coronal, axial, sagittal, and 3D images showing a baclofen-induced increase in right dIPFC cerebral blood flow (CBF) in the brain at rest, $Z=2.95, p=0.021$. (B) The dIPFC increase in the brain at rest predicted a decrease in the neural response to SCs in the ventral medial prefrontal cortex, $\mathrm{Z}=4.33$, $p_{\text {FWEcorr }}=$ 0.002 (top 3D image) and the left ventral anterior insula, $\mathrm{Z}=3.85$, $p_{\text {FWEcorr }}=0.002$ (bottom 3D image). Coordinates are in MNI space. Clusters are defined via whole-brain voxelwise analysis. Values are family-wise error (FWE) corrected. 
and an interaction effect between timepoint and group $\beta=$ $1.36, \mathrm{SE}=0.68, p=0.047$. Post-hoc $t$ tests revealed that this effect was driven by a reduction in SC-elicited craving in baclofen participants from T1 to T2, $t=2.90, p<0.01$ (Fig. 2), whereas there was no change in placebo participants between timepoints, $t=-0.09, p=0.93$.

\section{Imaging Results}

All imaging results can be viewed in detail at ArielKetcherside.com. For our first hypothesis, that baclofen would increase resting brain activity in cognitive control regions, we performed a full factorial model in SPM12. This showed a timepoint-by-group interaction such that $B A C$ participants had greater resting activation in the right dIPFC at $\mathrm{T} 2$ and compared to $\mathrm{PBO}(\mathrm{Z}=2.78$, SVC $p_{\text {FWEcorr }}=0.03$, Fig. 3A, see S1 for boxplot). There was no effect of timepoint on resting state $\mathrm{CBF}$ in our other ROls, the ACC or the left dIPFC in BAC.

Given our second hypothesis, that baclofen-induced change in CBF within our ROls at rest would associate with a decrease in the reward response during SC exposure, we extracted the values from the ROI wherein we saw an effect, the right dIPFC (calculated as T2-T1) and used them as covariates in the SC T2 model, as previously described [26]. We found that the baclofen-induced increase in right dIPFC at rest was associated with a decrease in neural response in the $\operatorname{vmPFC}\left(Z=4.33, p_{\text {FWEcorr }}=\right.$ $0.002)$ and left avInsula $\left(Z=3.85, \operatorname{SVC} p_{\text {FWEcorr }}=0.002\right)$ during SC exposure (Fig. 3B).

A

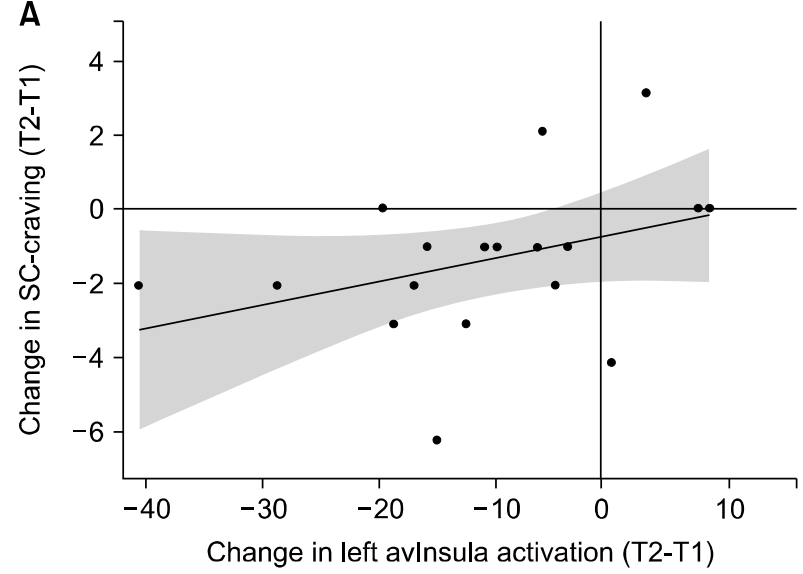

\section{Post-hoc Brain-behavior Correlations}

We performed a post-hoc analysis to determine if baclofen-induced changes in neural response to SCs correlated with subjective reports of SC-induced craving. First, we performed a full factorial analysis to identify a stimulus (SCs and non-SCs)-by-timepoint (T1 and T2) interaction effect on neural changes in SC response during treatment in both the baclofen and placebo groups. We then extracted the average value from a $5 \mathrm{~mm}$ sphere surrounding the peak voxel of the SC ROIs and examined whether these values correlated with the magnitude of change in SC-induced ratings for craving from $\mathrm{T} 1$ to $\mathrm{T} 2$. We observed a trend toward correlation between the magnitude of the decrease in SC-craving and the magnitude of the decrease in the left avInsula from $\mathrm{T} 1$ to $\mathrm{T} 2(-38,14,-16)$, $r=0.36, p=0.06$ and in the $\operatorname{vmPFC}(4,22,-14), r=0.35$, $p=0.07$, the two regions previously identified in our testing of hypothesis 2. While these do not pass the "bright-line fallacy" threshold of significance [58], or the Šidák correction for multiple comparisons that would be most prudent for interpretation as stand-alone results $\left(p_{\text {adjusted }}<0.03\right)[53,54,59]$, we present them here for interpretation in the context of our overall results. While further examination is necessary, these preliminary findings suggest that, the more BAC participants exhibited reduced activation in limbic regions, which have been shown throughout the literature to be associated with craving, the less SC-craving they reported (Fig. 4). The same analysis in PBO participants showed no correlations.

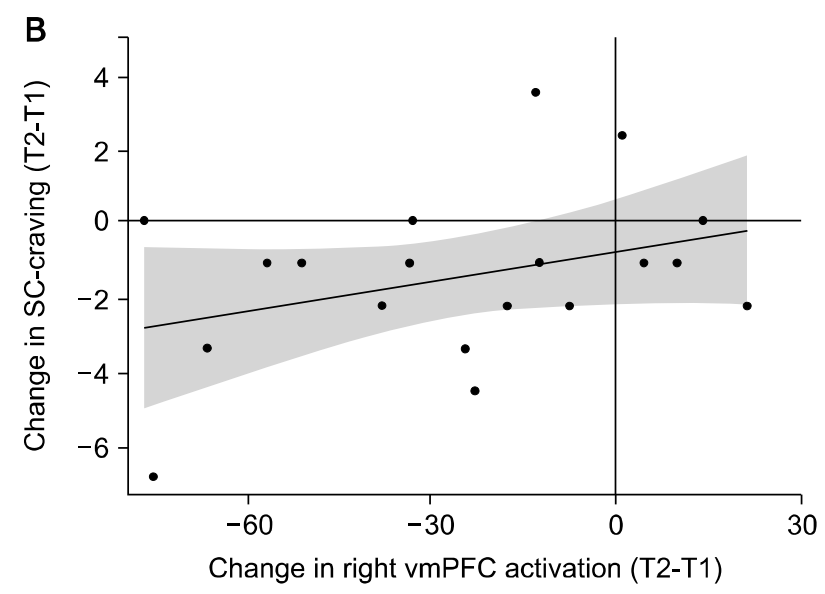

Fig. 4. Baclofen's effects on brain response to SCs trends toward correlation with SC-elicited craving. (A) The magnitude of decrease in SC-induced craving (T2-T1) trended toward correlation with the magnitude of decrease in left avlnsula activation (T2-T1), MNI coordinates $(-38,14,-16), r=$ $0.36, p=0.06$, as well as (B) the magnitude of decrease in the vmPFC, MNI coordinates $(4,22,-14), r=0.35, p=0.07$.

$\mathrm{SC}$, smoking cue; T1, before baclofen; T2, during treatment with baclofen; avInsula, anterior ventral insula; vmPFC, ventromedial prefrontal cortex. 
Additional post-hoc analyses on this data set are provided at ArielKetcherside.com.

\section{DISCUSSION}

We examined baclofen's effects on the resting brain in a priori cognitive control regions (ACC and dIPFC) and relationships between baclofen-induced resting changes and response to SCs in reward regions (vmPFC, VS, avInsula). Results indicate that baclofen increased resting right dIPFC activation, which was associated with a decrease in left avInsula and vmPFC SC-elicited activation. Further, while both groups reported SC-elicited craving prior to medication (T1), only baclofen-treated subjects showed reduced SC-elicited craving during treatment (T2). Our findings align well with those of Holla and colleagues [24] who showed that baclofen increased activity in the dIPFC and decreased activity in the insula in AUD patients during alcohol cue exposure that subsequently predicted time to relapse, suggesting a common mechanism for mitigating craving across substances of abuse.

Our finding that baclofen increased dIPFC activation at rest replicates our previous findings [25] and is consistent with literature implicating the dIPFC in recovery from substance use disorders [24,60-62]. The prevailing theory uniting these studies postulates that increased resting activity in the dIPFC can improve individuals' ability to mitigate conditioned responses that lead to relapse. The dIPFC is a hub of inhibitory control within the executive control network [63], and during SC-reactivity, it integrates cue perception, craving, and behavior planning to seek or avoid SCs [64]. Thus, the baclofen-induced increase in resting dIPFC observed here could indicate a similar increase in tonic executive control, enabling participants to self-regulate reactivity to SCs.

When included as a covariate in the model assessing SC-reactivity, the BAC-induced increase in resting dIPFC activation predicted a decrease in left avInsula activity in response to SCs, indicating that, the greater the tonic dIPFC activation, the less the avInsula responded to SCs. The insula integrates sensory, salience, and executive control networks, and the avinsula is associated with subjective "urge" for drugs in response to drug cues [9,29,64-68]. Janes et al. have shown that avInsula activation in response to SCs predicted relapse during subsequent smoking cessation attempts $[11,12]$ and that de- creased anterior insula-dlPFC functional connectivity during SC exposure predicts relapse [12]. In line with this work, our findings suggest that the greater the tonic activation of the dIPFC, the less the avInsula responds to SCs, which could potentially prevent relapse. Associations between the left avInsula and craving have been observed repeatedly in the SC literature, however the right insula has also been implicated, and thus the laterality of our results should be addressed $[11,12]$. One such explanation lies in previous work suggesting that the left avInsula responds more to positive stimuli than the right [69]. As our SCs are highly appetitive, the left avInsula may have been more engaged during SC exposure prior to medication, such that baclofen's effects are observable to a greater extent on the left side. Baclofen's modulation of the dIPFC was also associated with a decrease in vmPFC response to $\mathrm{SCs}$. This is congruent with literature showing that vmPFC activation during SC exposure is associated with craving, while its reduced activity is associated with smoking cessation interventions and reduced craving [26,30,70]. Collectively, results suggest a mechanism by which baclofen may blunt SC-responsivity, and thereby prevent SC-induced relapse.

Additionally, post-hoc analyses in baclofen participants showed that the magnitude of baclofen-induced decreases in neural response to SCs in the avlnsula and vmPFC (T1-T2) trended toward correlation with the magnitude of the decrease in subjective reports of SC-induced craving (T1-T2). In other words, the more BAC participants exhibited reduced activation in limbic regions, which have been established within the literature as mechanisms of craving, the less SC-craving they reported. While these correlations were small and only trended toward statistical significance, we note that patterns in the data are notable, given the small range of possible answers to the craving questionnaire $(1-7)$ and the ensuing double subtraction of the scores. Given that these results link our imaging and behavioral findings, they further support our hypothesis for the proposed mechanism of baclofen in SC-craving reduction. Furthermore, they are similar to our previous results [26]. As such, this relationship should be examined further in future work with a more sensitive measurement.

We did not observe baclofen-induced increases in the left dIPFC at rest. Evidence for functional heterogeneity between the right and left dIPFC indicates the right is in- 
volved in hierarchical evaluation, while the left is involved in the evaluation of possible outcomes [71]. Thus, tonic orientation toward identifying potentially relevant stimuli at rest [72], without the need to evaluate potential outcomes, may have engaged the right dIPFC in our study. Thus, lateralization should be examined in future work.

Baclofen's effects did not extend to the VS in the current study. This could be due to individual heterogeneity within our sample, including sex and/or genetic differences $[56,73]$, and the inclusion of non-SC vulnerable individuals, all of which we are underpowered to examine. These null findings underscore the heterogeneity in response to baclofen, and the importance of differentiating SC-vulnerable individuals from other treatment seekers.

To date, most studies of baclofen for SUDs are for AUD, with equivocal results [74-76], perhaps partially because potential heterogeneity in treatment response has not been examined. To date, three AUD studies have included neuroimaging during alcohol cue exposure $[21,22,24]$, with modest samples and mixed findings, however, they all emphasize (1) that baclofen's mechanism of action may be through blunting cue reactivity and that (2) more work is needed to understand this mechanism. Logge et al. particularly showed a dose-response effect such that a higher dose of baclofen $(75 \mathrm{mg} /$ day) reduced heavy drinking days compared to a lower dose (30 mg/day), which also warrants consideration in future studies.

Additional smoking-cessation and relapse-prevention medications are necessary for smokers trying to quit, as the most commonly-prescribed agent, varenicline, does not help the majority of patients to whom it is prescribed $[17,77]$. Our group previously showed that the mechanism by which varenicline reduces SC-reactivity is through an increase in resting lateral OFC activation, which predicts a decrease in SC-response in the medial OFC and ventral striatum [26]. Contrasted with the regions affected by baclofen in this study, this differential neural response to varenicline suggests that the medications have different mechanisms of action. Notably, baclofen, but not varenicline patients show a reduction in avInsula response to smoking cues, and the avInsula is well-established hub of interoception and craving $[11,66,68]$. In this vein, one might speculate that varenicline's limited effectiveness may be because it does not directly block SC-reactivity: in all previous varenicline studies examining SC-reactivity, participants continued to smoke during treatment, before assessment $[26,78,79]$. Perhaps the repeated blockade of nicotine reward while smoking extinguishes conditioned associations between the cues and cigarettes. Thus, as Brandon and colleagues explicitly suggest, the observed blockade of SC-induced craving could occur through temporary SC-reward extinction [78]. Thus, varenicline's action to block reinforcement suggests it may be more helpful for abstinence initiation in those whose vulnerabilities include avoidance of withdrawal and reward reinforcement [16], while baclofen may act as a relapseprevention agent in a cue-vulnerable subgroup, abstinent at treatment initiation. This previously unaddressed emphasis on the potential use of baclofen for relapse-prevention rather than abstinence-initiation could partially explain the discordant literature. As such, future studies may find it useful to design studies that explicitly test the usefulness of medications in abstinence-initiation and/or relapse prevention to address individual vulnerabilities.

Baclofen has several inherent advantages for use as a medication for smoking cessation. It is currently approved for muscle spasticity by the United States Food and Drug Administration [80], has a history of safety, is not abused, and its generally benign adverse effects, when present, are well-documented [81]. It is available in generic form and is cost-effective. Its short half-life ( 4 hours) suits its use as a relapse prevention medication on an as-needed basis: as SCs are often context-dependent (e.g., being surrounded by smokers at an event), an individual could take baclofen prospectively, on an as-needed basis. Thus, baclofen is affordable and carries minimal risk, with an acute mechanism of action precluding the need for daily dosage. Although baclofen's effectiveness as a smoking cessation aid was not tested here, it has shown promise in the few placebo-controlled studies that have tested it. In a paradigm similar to the current study, using the same dose of baclofen and setting the Quit Date to occur after 3 weeks of study participation, baclofen reduced CPD [82]. In another study conducted in alcohol dependent smokers seeking treatment for both, baclofen increased the number of days of abstinence of co-use [83]. While both of these preliminary studies show proof of concept for further clinical examination in treatment-seeking cigarette smokers baclofen's efficacy has been challenged [76]. An additional factor that may underlie the mixed therapeutic profile of baclofen is individual response to medication. While some studies show that low doses ( $\sim 30 \mathrm{mg}$ per day) 
are effective other studies show a dose response effect with higher doses showing more effectiveness [22]. Marsot et al. [84] recently identified a potential mitigating factor that may provide some insight. In this pharmacokinetic study the plasma concentration of baclofen showed marked interpatient variability that remained unexplained by a multitude of potential confounding factors.

\section{Limitations and Future Directions}

Although this study is strengthened by its within-subjects, placebo-controlled, longitudinal design, it has limitations. First, the modest sample size precludes our ability to examine heterogeneity in response to SCs or to baclofen that may be introduced by additional factors such as individual pharmacokinetics [84] that might be affected by sex, genetics, and hormonal variability $[40,73]$. Further, to heighten homogeneity in brain response, a particular design feature was to minimize changes in smoking behavior across participants; participants were asked to 'smoke as usual' until after the on-medication testing day and smoked to satiety immediately before both test days, which precluded the ability to study its potential effectiveness as a relapse prevention agent. Despite this, the literature to date indicates that baclofen does not block reinforcement while smoking, but rather influences reward circuitry $[20,85]$. Nonetheless future studies should examine baclofen's ability to block SC-reactivity explicitly in abstinent smokers.

\section{Conclusions}

To date, the majority of smokers remain unaided by first line available smoking cessation medications that act to mitigate withdrawal and block reinforcement. As SC reactivity is a known relapse predictor, additional treatment strategies that specifically target SC reactivity are crucial. Our results suggest that the GABA-B agonist, baclofen, mitigates the enhanced reward response to $\mathrm{SCs}$, and that this occurs through an increase in tonic activation of the dIPFC, an executive control region. This mechanism is congruent with the theory gaining recognition in the field, that strengthening tonic executive functioning facilitates recovery from SUDs. Finally, the variable effectiveness of baclofen for SUDs documented to date may actually indicate a difference in cue-vulnerability vs. reward-sensitivity in treatment-seeking individuals. Thus, baclofen may be most effective in facilitating relapse prevention in cue vul- nerable smokers who have already quit.

\section{- Acknowledgments}

The authors wish to thank Santos B. Murty and Murty Pharmaceuticals, Inc. for providing the medication; the Investigational Drug Service at the University of Pennsylvania for management of study medication; Melanie Maron, MS for coordinating the study; the clinical staff at the University of Pennsylvania Center for the Studies of Addiction, for conducting physical and psychiatric evaluations and medication monitoring; and the MRI technicians at the Hospital of the University of Pennsylvania for conducting the scanning sessions.

\section{- Conflicts of Interest}

No potential conflict of interest relevant to this article was reported.

\section{Author Contributions}

Conceptualization: Teresa Franklin. Data acquisition: Nathan Hager, Chaela Nutor, Nathanial Spilka. Formal analysis: Ariel Ketcherside, Kanchana Jagannathan. Analysis support: Sudipto Dolui, Hengyi Rao. Funding: R01 DA0303994 (Teresa Franklin), R01 DA029845 (Teresa Franklin), K23AA023894 (Reagan Wetherill), R01DA040670 (Reagan Wetherill), R03 AG063213 (Sudipto Dolui). Writing-original draft: Ariel Ketcherside. Writing-review: All authors.

\section{ORCID}

Ariel Ketcherside https://orcid.org/0000-0003-1885-3581 Kanchana Jagannathan

https://orcid.org/0000-0002-8314-0898

Sudipto Dolui https://orcid.org/0000-0002-9035-3795

Nathan Hager https://orcid.org/0000-0003-2083-7337

Nathaniel Spilka https://orcid.org/0000-0002-3319-4919

Chaela Nutor https://orcid.org/0000-0002-5469-2802

Hengyi Rao https://orcid.org/0000-0003-2735-2500

Teresa Franklin https://orcid.org/0000-0002-1430-2312

Reagan Wetherill https://orcid.org/0000-0002-1991-6292

\section{REFERENCES}

1. Commar A, Prasad VK, d'Espaignet ET, Wolfenden L. WHO global report on trends in prevalence of tobacco smoking 2000-2025. Geneva:World Health Organization;2018.

2. Babb S, Malarcher A, Schauer G, Asman K, Jamal A. Quitting 
smoking among adults - United States, 2000-2015. Morbidity and Mortality Weekly Report (MMWR) [Internet]. Centers for Disease Control and Prevention; 2017 [cited at 2019 Mar 1]. Available from: https://www.cdc.gov/mmwr/volumes/65/wr/ mm6552a1.htm.

3. García-Rodríguez O, Secades-Villa R, Flórez-Salamanca L, Okuda M, Liu SM, Blanco C. Probability and predictors of relapse to smoking: results of the National Epidemiologic Survey on Alcohol and Related Conditions (NESARC). Drug Alcohol Depend 2013;132:479-485.

4. Perkins KA. Does smoking cue-induced craving tell us anything important about nicotine dependence? Addiction 2009; 104:1610-1616.

5. Carter BL, Tiffany ST. Cue-reactivity and the future of addiction research. Addiction 1999;94:349-351.

6. Drummond DC. What does cue-reactivity have to offer clinical research? Addiction 2000;95 Suppl 2:S129-S144.

7. Tiffany ST, Wray J. The continuing conundrum of craving. Addiction 2009;104:1618-1619.

8. Bedi G, Preston KL, Epstein DH, Heishman SJ, Marrone GF, Shaham Y, et al. Incubation of cue-induced cigarette craving during abstinence in human smokers. Biol Psychiatry 2011; 69:708-711.

9. Franklin TR, Wang Z, Wang J, Sciortino N, Harper D, Li Y, et al. Limbic activation to cigarette smoking cues independent of nicotine withdrawal: a perfusion fMRI study. Neuropsychopharmacology 2007;32:2301-2309.

10. Conklin CA, Vella EJ, Joyce CJ, Salkeld RP, Perkins KA, Parzynski CS. Examining the relationship between cue-induced craving and actual smoking. Exp Clin Psychopharmacol 2015;23:90-96

11. Janes AC, Gilman JM, Radoman M, Pachas G, Fava M, Evins $\mathrm{AE}$. Revisiting the role of the insula and smoking cue-reactivity in relapse: a replication and extension of neuroimaging findings. Drug A/cohol Depend 2017;179:8-12.

12. Janes AC, Pizzagalli DA, Richardt S, deB Frederick B, Chuzi $S$, Pachas $\mathrm{G}$, et al. Brain reactivity to smoking cues prior to smoking cessation predicts ability to maintain tobacco abstinence. Biol Psychiatry 2010;67:722-729.

13. Versace F, Engelmann JM, Robinson JD, Jackson EF, Green CE, Lam CY, et al. Prequit fMRI responses to pleasant cues and cigarette-related cues predict smoking cessation outcome. Nicotine Tob Res 2014;16:697-708.

14. Wilkes S. The use of bupropion SR in cigarette smoking cessation. Int J Chron Obstruct Pulmon Dis 2008;3:45-53.

15. Mihalak KB, Carroll FI, Luetje CW. Varenicline is a partial agonist at alpha4beta2 and a full agonist at alpha7 neuronal nicotinic receptors. Mol Pharmacol 2006;70:801-805.

16. Rollema H, Chambers LK, Coe JW, Glowa J, Hurst RS, Lebel LA, et al. Pharmacological profile of the alpha4beta2 nicotinic acetylcholine receptor partial agonist varenicline, an effective smoking cessation aid. Neuropharmacology 2007; 52:985-994.
17. Ebbert JO, Hughes JR, West RJ, Rennard SI, Russ C, McRae TD, et al. Effect of varenicline on smoking cessation through smoking reduction: a randomized clinical trial. JAMA 2015; 313:687-694.

18. Fadda P, Scherma M, Fresu A, Collu M, Fratta W. Baclofen antagonizes nicotine-, cocaine-, and morphine-induced dopamine release in the nucleus accumbens of rat. Synapse 2003; 50:1-6.

19. Brebner K, Childress AR, Roberts DC. A potential role for $G A B A(B)$ agonists in the treatment of psychostimulant addiction. A/cohol Alcohol 2002;37:478-484.

20. Fattore L, Spano MS, Cossu G, Scherma M, Fratta W, Fadda P. Baclofen prevents drug-induced reinstatement of extinguished nicotine-seeking behaviour and nicotine place preference in rodents. Eur Neuropsychopharmacol 2009;19:487-498.

21. Beck A, Pelz P, Lorenz RC, Charlet K, Geisel O, Heinz A, et al. Effects of high-dose baclofen on cue reactivity in alcohol dependence: a randomized, placebo-controlled pharmaco-fMRI study. Eur Neuropsychopharmacol 2018;28:1206-1216.

22. Logge WB, Morris RW, Baillie AJ, Haber PS, Morley KC. Baclofen attenuates $\mathrm{FMRI}$ alcohol cue reactivity in treatmentseeking alcohol dependent individuals. Psychopharmacology (Berl) 2019. doi: 10.1007/s00213-019-05192-5. [Epub ahead of print]

23. Young KA, Franklin TR, Roberts DC, Jagannathan K, Suh JJ, Wetherill RR, et al. Nipping cue reactivity in the bud: baclofen prevents limbic activation elicited by subliminal drug cues. J Neurosci 2014;34:5038-5043.

24. Holla B, Karthik S, Biswal J, Viswanath B, Jayarajan D, Bharath $\mathrm{RD}$, et al. Brain functional magnetic resonance imaging cue-reactivity can predict baclofen response in alcohol use disorders. Clin Psychopharmacol Neurosci 2018;16:290-301.

25. Franklin TR, Wang Z, Sciortino N, Harper D, Li Y, Hakun J, et al. Modulation of resting brain cerebral blood flow by the GABA B agonist, baclofen: a longitudinal perfusion $\mathrm{AMRI}$ study. Drug A/cohol Depend 2011;117:176-183.

26. Franklin T, Wang Z, Suh JJ, Hazan R, Cruz J, Li Y, et al. Effects of varenicline on smoking cue-triggered neural and craving responses. Arch Gen Psychiatry 2011;68:516-526.

27. McHugh MJ, Gu H, Yang Y, Adinoff B, Stein EA. Executive control network connectivity strength protects against relapse to cocaine use. Addict Biol 2017;22:1790-1801.

28. Zilverstand A, Huang AS, Alia-Klein N, Goldstein RZ. Neuroimaging impaired response inhibition and salience attribution in human drug addiction: a systematic review. Neuron 2018;98:886-903.

29. Franklin TR, Jagannathan K, Wetherill RR, Johnson B, Kelly S, Langguth J, et al. Influence of menstrual cycle phase on neural and craving responses to appetitive smoking cues in naturally cycling females. Nicotine Tob Res 2015;17:390-397.

30. Dumais KM, Franklin TR, Jagannathan K, Hager N, Gawrysiak $\mathrm{M}$, Betts J, et al. Multi-site exploration of sex differences in brain reactivity to smoking cues: consensus across sites and 
methodologies. Drug A/cohol Depend 2017;178:469-476.

31. Fagerstrom KO, Schneider NG. Measuring nicotine dependence: a review of the Fagerstrom Tolerance Questionnaire. J Behav Med 1989;12:159-182.

32. Sheehan DV, Lecrubier $Y$, Sheehan KH, Amorim P, Janavs J, Weiller E, et al. The Mini-International Neuropsychiatric Interview (M.I.N.I.): the development and validation of a structured diagnostic psychiatric interview for DSM-IV and ICD-10. J Clin Psychiatry 1998;59 Suppl 20:22-33;quiz 34-57.

33. Wechsler D. WASI, Wechsler abbreviated scale of intelligence. San Antonio:Psychological Corporation; 1999.

34. Dill T. Contraindications to magnetic resonance imaging: non-invasive imaging. Heart 2008;94:943-948.

35. Byrne JEM, Hughes ME, Rossell SL, Johnson SL, Murray G. Time of day differences in neural reward functioning in healthy young men. J Neurosci 2017;37:8895-8900.

36. Wu WC, Fernández-Seara M, Detre JA, Wehrli FW, Wang J. A theoretical and experimental investigation of the tagging efficiency of pseudocontinuous arterial spin labeling. Magn Reson Med 2007; 58:1020-1027.

37. Hermes M, Hagemann D, Britz P, Lieser S, Rock J, Naumann $\mathrm{E}$, et al. Reproducibility of continuous arterial spin labeling perfusion MRI after 7 weeks. MAGMA 2007;20:103-115.

38. Benowitz NL, Gourlay SG. Cardiovascular toxicity of nicotine: implications for nicotine replacement therapy. I Am Coll Cardiol 1997;29:1422-1431.

39. Wilson SJ, Sayette MA, Fiez JA, Brough E. Carry-over effects of smoking cue exposure on working memory performance. Nicotine Tob Res 2007;9:613-619.

40. Wetherill RR, Young KA, Jagannathan K, Shin J, O'Brien CP, Childress AR, et al. The impact of sex on brain responses to smoking cues: a perfusion fMRI study. Biol Sex Differ 2013; 4:9.

41. Wang Z, Aguirre GK, Rao H, Wang J, Fernández-Seara MA, Childress AR, et al. Empirical optimization of ASL data analysis using an ASL data processing toolbox: ASLtbx. Magn Reson Imaging 2008;26:261-269.

42. FMRIB Analysis Group. The manual of FMRIB software library. Oxford:FMRIB Analysis Group;2018.

43. MathWorks Inc. The manual of Statistics and machine learning toolbox-release 2016a. Natick:MathWorks Inc;2016.

44. Wang Z. Improving cerebral blood flow quantification for arterial spin labeled perfusion MRI by removing residual motion artifacts and global signal fluctuations. Magn Reson Imaging 2012;30:1409-1415.

45. Greve DN, Fischl B. Accurate and robust brain image alignment using boundary-based registration. Neuroimage 2009; 48:63-72.

46. Dolui S, Vidorreta M, Wang Z, Nasrallah IM, Alavi A, Wolk DA, et al. Comparison of PASL, PCASL, and background-suppressed 3D PCASL in mild cognitive impairment. Hum Brain Mapp 2017;38:5260-5273.

47. Alsop DC, Detre JA, Golay X, Günther M, Hendrikse J,
Hernandez-Garcia L, et al. Recommended implementation of arterial spin-labeled perfusion MRI for clinical applications: a consensus of the ISMRM perfusion study group and the European consortium for ASL in dementia. Magn Reson Med 2015;73:102-116.

48. Dolui S, Wang Z, Shinohara RT, Wolk DA, Detre JA; Alzheimer's Disease Neuroimaging Initiative. Structural Correlation-based Outlier Rejection (SCORE) algorithm for arterial spin labeling time series. J Magn Reson Imaging 2017;45:1786-1797.

49. Dolui S, Wolk DA, Detre JA. SCRUB: a structural correlation and empirical robust bayesian method for ASL data. In: ISMRM 25th Annual Meeting and Exhibition; May 7-8, 2016; Singapore.

50. Dolui S, Wolf R, Nabavizadeh SA, Wolk DA, Detre JA. Automated quality evaluation index for 2D ASL CBF maps. In: ISMRM 25th Annual Meeting and Exhibition; Apr 22-27, 2017; Hawaii, USA.

51. R Core Team. $R$ : a language and environment for statistical computing [Internet]. Vienna: $R$ Foundation for Statistical Computing; 2017. [cited at 2017 Oct 16]. Available from: https://www.r-project.org.

52. Bredlau AL, Harel BT, McDermott MP, Dworkin RH, Korones DN, Dolan JG, et al. Neurocognitive changes after sustained ketamine administration in children with chronic pain. J Palliat Care Med 2015;5:215.

53. Abdi $\mathrm{H}$. The Bonferonni and Šidák corrections for multiple comparisons. 2007. 9 p.

54. Blakesley RE, Mazumdar S, Dew MA, Houck PR, Tang G, Reynolds CF 3rd, et al. Comparisons of methods for multiple hypothesis testing in neuropsychological research. Neuropsychology 2009;23:255-264.

55. Lancaster JL, Laird AR, Eickhoff SB, Martinez MJ, Fox PM, Fox PT. Automated regional behavioral analysis for human brain images. Front Neuroinform 2012;6:23.

56. Wetherill RR, Jagannathan K, Hager N, Childress AR, Franklin TR. Sex differences in associations between cannabis craving and neural responses to cannabis cues: Implications for treatment. Exp Clin Psychopharmacol 2015;23:238-246.

57. Dumais KM, Franklin TR, Jagannathan K, Hager N, Gawrysiak $\mathrm{M}$, Betts J, et al. Multi-site exploration of sex differences in brain reactivity to smoking cues: consensus across sites and methodologies. Drug Alcohol Depend 2017;178:469-476.

58. Wasserstein RL, Lazar NA. The ASA statement on p-values: context, process, and purpose. Am Stat 2016;70:129-133.

59. Lindquist MA, Mejia A. Zen and the art of multiple comparisons. Psychosom Med 2015;77:114-125.

60. Zelle SL, Gates KM, Fiez JA, Sayette MA, Wilson SJ. The first day is always the hardest: Functional connectivity during cue exposure and the ability to resist smoking in the initial hours of a quit attempt. Neuroimage 2017; 151:24-32.

61. Schluter RS, Daams JG, van Holst RJ, Goudriaan AE. Effects of non-invasive neuromodulation on executive and other cognitive functions in addictive disorders: a systematic review. 
Front Neurosci 2018;12:642.

62. Karch S, Paolini M, Gschwendtner S, Jeanty H, Reckenfelderbäumer A, Yaseen O, et al. Real-time fMRI neurofeedback in patients with tobacco use disorder during smoking cessation: functional differences and implications of the first training session in regard to future abstinence or relapse. Front Hum Neurosci 2019;13:65.

63. Clarke PJ, Browning M, Hammond G, Notebaert L, MacLeod C. The causal role of the dorsolateral prefrontal cortex in the modification of attentional bias: evidence from transcranial direct current stimulation. Biol Psychiatry 2014;76:946-952.

64. McBride D, Barrett SP, Kelly JT, Aw A, Dagher A. Effects of expectancy and abstinence on the neural response to smoking cues in cigarette smokers: an fMRI study. Neuropsychopharmacology 2006;31:2728-2738.

65. Lee JH, Lim Y, Wiederhold BK, Graham SJ. A functional magnetic resonance imaging (FMRI) study of cue-induced smoking craving in virtual environments. Appl Psychophysiol Biofeedback 2005;30:195-204.

66. Naqvi NH, Bechara A. The insula and drug addiction: an interoceptive view of pleasure, urges, and decision-making. Brain Struct Funct 2010;214:435-450.

67. Claus ED, Blaine SK, Filbey FM, Mayer AR, Hutchison KE. Association between nicotine dependence severity, BOLD response to smoking cues, and functional connectivity. Neuropsychopharmacology 2013;38:2363-2372.

68. Janes AC, Ross RS, Farmer S, Frederick BB, Nickerson LD, Lukas SE, et al. Memory retrieval of smoking-related images induce greater insula activation as revealed by an fMRI-based delayed matching to sample task. Addict Biol 2015;20:349-356.

69. Duerden EG, Arsalidou M, Lee M, Taylor MJ. Lateralization of affective processing in the insula. Neuroimage 2013;78:159175.

70. Janes AC, Farmer S, Frederick Bd, Nickerson LD, Lukas SE. An increase in tobacco craving is associated with enhanced medial prefrontal cortex network coupling. PLoS One 2014;9: e88228.

71. Kaller CP, Rahm B, Spreer J, Weiller C, Unterrainer JM. Dissociable contributions of left and right dorsolateral prefrontal cortex in planning. Cereb Cortex 2011;21:307-317.

72. DeWitt SJ, Ketcherside A, McQueeny TM, Dunlop JP, Filbey FM. The hyper-sentient addict: an exteroception model of addiction. Am J Drug Alcohol Abuse 2015:41:374-381.

73. Franklin TR, Lohoff FW, Wang Z, Sciortino N, Harper D, Li Y, et al. DAT genotype modulates brain and behavioral responses elicited by cigarette cues. Neuropsychopharmacology 2009;34:717-728.

74. Addolorato G, Leggio L, Ferrulli A, Cardone S, Bedogni G,
Caputo F, et al. Dose-response effect of baclofen in reducing daily alcohol intake in alcohol dependence: secondary analysis of a randomized, double-blind, placebo-controlled trial. Alcohol Alcohol 2011;46:312-317.

75. Agabio R, Sinclair JM, Addolorato G, Aubin HJ, Beraha EM, Caputo F, et al. Baclofen for the treatment of alcohol use disorder: the Cagliari Statement. Lancet Psychiatry 2018;5:957-960.

76. de Beaurepaire R, Sinclair JMA, Heydtmann M, Addolorato G, Aubin HJ, Beraha EM, et al. The use of baclofen as a treatment for alcohol use disorder: a clinical practice perspective. Front Psychiatry 2019;9:708.

77. West R, Baker CL, Cappelleri JC, Bushmakin AG. Effect of varenicline and bupropion SR on craving, nicotine withdrawal symptoms, and rewarding effects of smoking during a quit attempt. Psychopharmacology (Berl) 2008;197:371-377.

78. Brandon TH, Drobes DJ, Unrod M, Heckman BW, Oliver JA, Roetzheim RC, et al. Varenicline effects on craving, cue reactivity, and smoking reward. Psychopharmacology (Berl) 2011;218:391-403.

79. Ray LA, Lunny K, Bujarski S, Moallem N, Krull JL, Miotto K. The effects of varenicline on stress-induced and cue-induced craving for cigarettes. Drug A/cohol Depend 2013;131: 136-142.

80. Ghanavatian S, Derian A. Baclofen [Internet]. Treasure Island (FL): StatPearls Publishing; 2019 [cited at 2019 Apr 18]. Available from: http://www.ncbi.n/m.nih.gov/books/NBK526037/.

81. PubChem. Baclofen [Internet]. Bethesda: National Center for Biotechnology Information; 2018 [cited at 2018 Oct 1]. Available from: https://pubchem.ncbi.n/m.nih.gov/compound/ 2284.

82. Franklin TR, Harper D, Kampman K, Kildea-McCrea S, Jens W, Lynch KG, et al. The GABA B agonist baclofen reduces cigarette consumption in a preliminary double-blind placebocontrolled smoking reduction study. Drug A/cohol Depend 2009;103:30-36.

83. Leggio L, Zywiak WH, Edwards SM, Tidey JW, Swift RM, Kenna GA. A preliminary double-blind, placebo-controlled randomized study of baclofen effects in alcoholic smokers. Psychopharmacology (Berl) 2015;232:233-243.

84. Marsot A, Imbert B, Alvarez JC, Grassin-Delyle S, Jaquet I, Lançon C, et al. High variability in the exposure of baclofen in alcohol-dependent patients. Alcohol Clin Exp Res 2014;38: 316-321.

85. Westerink BH, Enrico P, Feimann J, De Vries JB. The pharmacology of mesocortical dopamine neurons: a dual-probe microdialysis study in the ventral tegmental area and prefrontal cortex of the rat brain. J Pharmacol Exp Ther 1998;285:143154. 